\title{
Hematuria in a Patient with
}

\section{Non-malignant Bladder}

\section{Nodules}

\author{
Terese S Bergheim ${ }^{1}$, Visal Nga1 ${ }^{1}$, Andrew C Burg ${ }^{1}$ and \\ Bahman Chavoshan ${ }^{1,2 *}$
}

${ }^{1}$ Department of Internal Medicine, St. Mary Medical Center, Long Beach, California, USA

Received: 26 March, 2019

Accepted: 11 April, 2020

Published: 13 April, 2020

*Corresponding author: Bahman Chavoshan, MD MAvMed, FCCP, Program Director, Associate Clinical Professor of Medicine, St. Mary UCLA Internal Medicine Residency Program, University of California, Los Angeles, USA, Tel: +1-562-491-9140; Fax: +1-562-4919146 E-mail:b.chavoshan@ucla.edu

Keywords: Malakoplakia; Hematuria; Bladder nodules ORCID: https://orcid.org/0000-0002-8860-3951

https://www. peertechz.com

\section{Check for updates}

${ }^{2}$ University of California, Los Angeles, USA

\section{Abstract}

Introduction: Gross hematuria in adults with resultant anemia is highly concerning for genitourinary malignancy. However, in rare instances, malakoplakia can mimic such malignancy. Malakoplakia is a benign granulomatous condition with malfunction of the phagolysosomal activity of macrophages and monocytes. This leads to formation of foamy histocytes with intracytoplasmic basophilic inclusions known as Michaelis-Gutmann bodies. These inclusion bodies are pathognomonic for the condition.

Case presentation: A 41-year-old female presented with spontaneous gingival bleed and painless gross hematuria for 2 days. Two weeks prior to the current admission she had been hospitalized with gastrointestinal bleed and gross hematuria with symptomatic anemia. The hematuria diminished during treatment with ceftriaxone. She was discharged with a course of cephalexin to treat complicated cystitis. On the subsequent admission, she denied taking any medications. Labs on

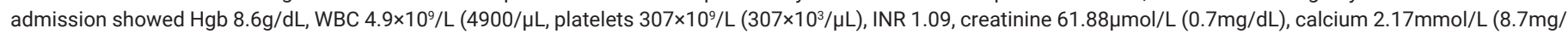
$\mathrm{dL}$ ). Cystoscopy showed several very abnormal appearing nodules present all over the bladder in a very unusual and abnormal fashion. Frozen section from the biopsy showed no evidence of malignancy but possible fungal infection. Final biopsy result showed malakoplakia, negative stains for acid fast bacilli and fungal organisms, and no evidence of tumor. Urine cultures for bacteria, fungus, acid fast bacilli were negative. Tissue culture grew light Enterococcus faecium, vancomycin resistant (VRE), sensitive to linezolid and quinopristin/dalfopristin, and light Candida glabrata. She was treated presumptively with ceftriaxone but switched to linezolid based on results from tissue cultures.

Discussion: The German scientists and physicians Michaelis and Gutmann first described Malakoplakia in 1902. Malakoplakia is a rare condition that most commonly affects the urinary tract and can mimic tumors. It is a benign condition characterized by a granulomatous process consisting of foamy histiocytes with basophilic Michaelis-Gutmann bodies. These basophilic inclusions consist of partially digested bacteria and calcium and iron deposits that result from a defective phagolysosomal activity in the macrophages. It is more commonly reported in patients with immunodeficiencies like HIV or in transplant patients. It is important to obtain a correct pathologic diagnosis in these cases given that it is a benign condition that mimics carcinoma. Treatment consists of a prolonged course of antibiotics. Bethanechol and ascorbic acid appear to improve cure rates.

\section{Introduction}

Gross hematuria in adults causing symptomatic anemia is highly concerning for malignancy. However, in rare instances, there are other mimics of bladder neoplasm.

Malakoplakia, is a benign granulomatous condition reflecting the malfunction of the phagolysosome activity of macrophages and monocytes. This leads to formation of foamy histiocytes with intracytoplasmic basophilic inclusions called Michaelis-Gutmann bodies, which are pathognomonic for the condition
This case presents a female with symptomatic anemia secondary to gross hematuria from multiple bladder nodules.

\section{Case presentation}

A 41-year-old female presented with gingival bleed and gross hematuria for 2 days. Two weeks prior, she had been hospitalized with GI bleed, gross hematuria and symptomatic anemia. The hematuria diminished during treatment with ceftriaxone for a suspected UTI. She was also found to have thickened and irregular bladder wall on abdominal CT. She was discharged home with a course of cephalexin 
to treat complicated cystitis. An outpatient cystoscopy was recommended to evaluate the irregular bladder wall thickening. It is unclear whether she completed her course of antibiotics, as she denied taking any medications when she was readmitted.

During the current admission, physical exam showed hypervascular and swollen gums, but was otherwise unremarkable. Admission hematology revealed $\mathrm{Hgb} 86 \mathrm{~g} / \mathrm{L}$ $(8.6 \mathrm{~g} / \mathrm{dL})$, WBC $4.9 \times 10^{9} / \mathrm{L}$, platelets $307 \times 10^{9} / \mathrm{L}$, and INR 1.09 . Serum biochemistry showed calcium $2.17 \mathrm{mmol} / \mathrm{L}(8.7 \mathrm{mg} / \mathrm{dL})$ and creatinine $61.88 \mu \mathrm{mol} / \mathrm{L}(0.7 \mathrm{mg} / \mathrm{dL})$. Iron was $342 \mathrm{mcg} /$ $\mathrm{dl}$ and ferritin $142 \mathrm{ng} / \mathrm{ml}$. Cystoscopy revealed a multitude of abnormal appearing nodules that were distributed diffusely in the bladder (Figures 1,2). Frozen sections from the biopsy did not show any malignancy but fungal infection could not be excluded. Final biopsy result showed malakoplakia (Figures 3-5). There was no evidence of malignancy and special stains for acid fast bacilli and fungal were negative. Urine cultures for bacteria, fungi and mycobacteria were negative. Tissue culture obtained from direct bladder biopsy grew Enterococcus faecium, Vancomycin Resistant (VRE), which was sensitive to linezolid as well as to quinupristin/dalfopristin. Light growth of Candida glabrata was also noted.

She was initially treated presumptively with ceftriaxone. Based on the in vitro sensitivities from the tissue culture, treatment was switched to linezolid. Her hematuria subsided. She was discharged home to complete a prolonged course of linezolid, with follow up arranged to monitor for medicationinduced hematologic toxicity.

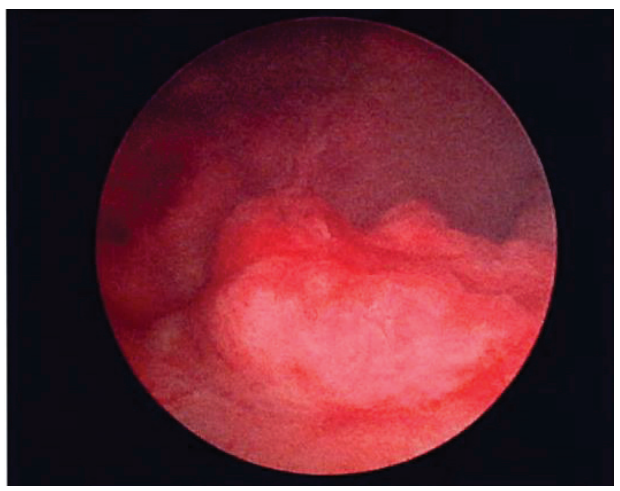

Figure 1: Bladder nodules as seen on cystoscopy (tangential view)

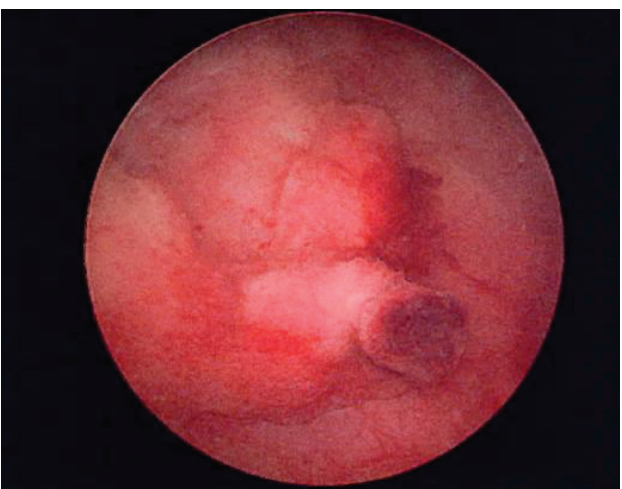

Figure 2: Bladder nodules (en face view)

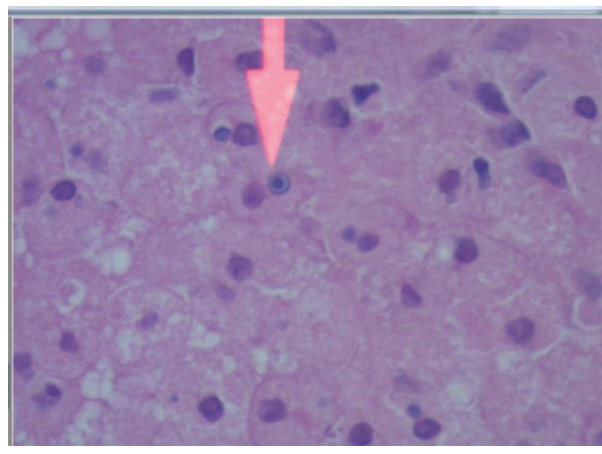

Figure 3: Histiocytes with Michaelis-Gutmann bodies (arrow) (H\&E stain)

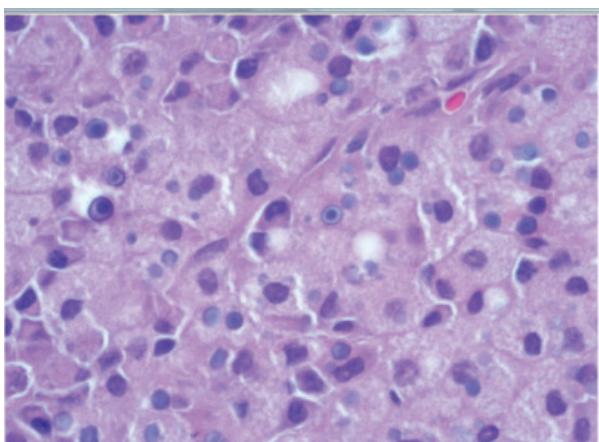

Figure 4: Bladder biopsy showing multiple MG-bodies. (H\&E stain).

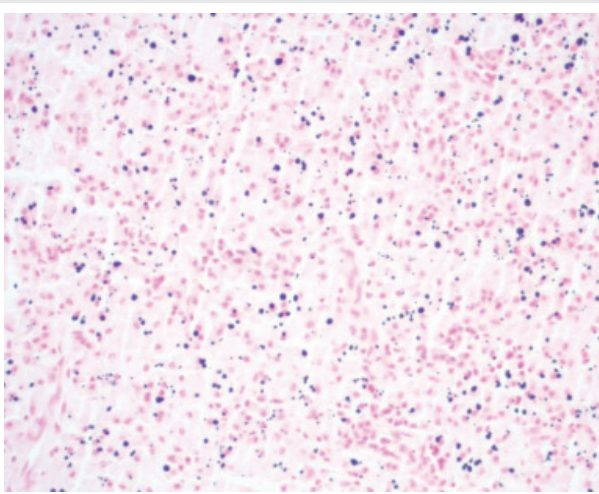

Figure 5: Black dots indicating cytoplasmic laminated mineralized concretions (Von Kossa stain)

\section{Discussion}

Malakoplakia is a rare, non-neoplastic condition that most commonly affects the urinary tract $(75 \%-80 \%$ of cases). Other sites have been reported including skin, CNS, tongue, lungs, gastrointestinal tract and bone. The female to male ratio is $4: 1$. The condition most commonly presents in the fourth and fifth decade of life. In $40 \%$ of the cases, malakoplakia is associated with immunodeficiency states, such as HIV/AIDS, malignancy, and post-transplantation.

DP von Hansemann named the condition malakoplakia based upon his observation of soft (malakos) plaques (plakos), Greek. On gross examination the lesions consist of yellow nodules and plaques that may mimic malignant tumors. On histopathology, a granulomatous process consisting of foamy histiocytes (Hansemann macrophages) with basophilic

Citation: Bergheim TS, Nga V, Burg AC, Chavoshan B (2020) Hematuria in a Patient with Non-malignant Bladder Nodules. Glob J Rare Dis 5(1): 007-009. DOI: https://dx.doi.org/10.17352/2640-7876.000021 
inclusions characterizes malakoplakia. In 1902, Michaelis and Gutmann (MG) first described these pathognomonic inclusions. Now named MG bodies, they consist of partially digested bacteria with calcium and iron deposits. These inclusions stain positive with von Kossa, Periodic Acid-Schiff (PAS) and Prussian blue stains.

MG bodies are the result of defective activity of macrophage phagolysosomes. While the exact underlying mechanism is unknown, an imbalance in intracellular cGMP/CAMP ratio is postulated to result in incomplete bacterial digestion. E. coli is the most commonly associated bacterium. Malakoplakia can also occur with other bacteria including Staphylococcus aureus, Mycobacterium tuberculosis, Klebsiella pneumoniae and Proteus spp. In the setting of HIV/AIDS, Rhodococcus equi has been frequently reported.

Malakoplakia is a benign, chronic condition that can be managed conventionally with prolonged antibiotic therapy to decrease the bacterial load. When the upper urinary tract and kidneys are involved, the clinical course can be more aggressive. Surgical treatment, such as debulking and ureteral stenting may be required.

Antibiotics with high intracellular concentration, including ciprofloxacin, rifampin, and trimethoprim/sulfamethoxazole, are considered first line agents. There is a case report of successful treatment of renal allograft-associated malakoplakia with a 13-month combination therapy consisting of faropenem and fosfomycin.

Some clinicians have added bethanechol and ascorbic acid as an adjunct to antibiotic therapy. In theory, these agents may enhance phagocytic activity by increasing intracellularly cGMP and reducing cAMP, respectively. The improved cGMP/ CAMP balance may result in improved intracellular bactericidal activity of the macrophages. Clinical results have been mixed [1-5].

\section{Conclusion}

This is a case of hematuria with an unusual etiology. On radiologic examination (CT scan) and on cystoscopy, a preliminary diagnosis of carcinoma of the bladder was strongly considered. However, the histopathologic changes seen on the bladder biopsy revealed malakoplakia, a non-neoplastic condition. This case also illustrates the usefulness of tissue cultures even when urine cultures are negative. In our patient, VRE was identified, and the symptoms of malakoplakia rapidly improved upon treatment with linezolid.

\section{References}

1. Long JP, Althausen AF (1989) Malacoplakia: A 25 Year Experience with Review of the Literature. J Urol 141: 1328-1331. Link: https://bit.ly/2VnniCK

2. Yousef GM, Naghibi B, Hamodat MM (2007) Malakoplakia Outside the Urinary Tract. Arch Pathol Lab Med 131: 297-300. Link: https://bit.ly/2Vj7dOo

3. Gupta R, Mahajan A, Atri S, Gupta CL (2013) Recurrent Painless Hematuria Secondary to Malacoplakia of the Urinary Bladder. A Case Report and Review of Literature. Urol J 10: 821-823. Link: https://bit.ly/2RxH5yd

4. Graves AL, Texler M, Manning L, Kulkarni H (2014) Successful treatment of rena allograft and bladder malakoplakia with minimization of immunosuppression and prolonged antibiotic therapy. Nephrology 19: 18-21. Link: https://bit.ly/3edkCAa

5. McCLure J (1983) Malakoplakia. J Pathology 140: 275-330. Link: https://bit.ly/2Ry9018

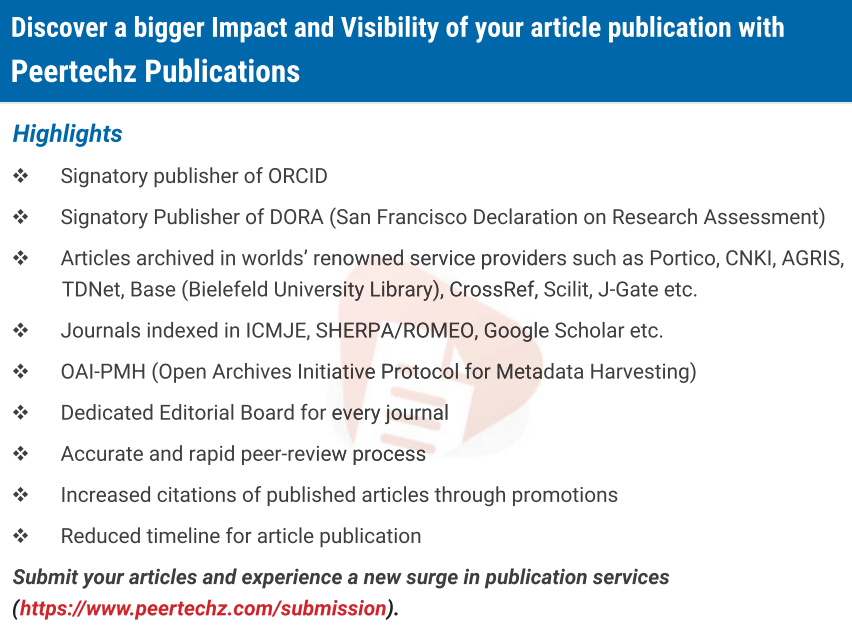

Peertechz journals wishes everlasting success in your every endeavours.

Copyright: ( 2020 Bergheim TS, et al. This is an open-access article distributed under the terms of the Creative Commons Attribution License, which permits unrestricted use, distribution, and reproduction in any medium, provided the original author and source are credited. 